\title{
Analysis of transients in a canal network
}

\author{
RAJEEV MISRA, M S MOHAN KUMAR and K SRIDHARAN \\ Department of Civil Engineering, Indian Institute of Science, Bangalore \\ 560012 , India
}

MS received 5 November 1990; revised 30 August 1991

\begin{abstract}
A generalised formulation of the mathematical model developed for the analysis of transients in a canal network, under subcritical flow, with any realistic combination of control structures and their multiple operations, has been presented. The model accounts for a large variety of control structures such as weirs, gates, notches etc. discharging under different conditions, namely submerged and unsubmerged. A numerical scheme to compute and approximate steady state flow condition as the initial condition has also been presented. The model can handle complex situations that may arise from multiple gate operations. This has been demonstrated with a problem wherein the boundary conditions change from a gate discharge equation to an energy equation and back to a gate discharge equation. In such a situation the wave strikes a fixed gate and leads to large and rapid fluctuations in both discharge and depth.
\end{abstract}

Keywords. Unsteady flow; open channel networks; canal transients.

\section{Introduction}

The analysis of transients in a canal network can play an important role in investigating the performance, operation and management of canal systems. A few models to simulate unsteady flow in channel networks have been presented in the past. Stoker (1957) made the first attempt to solve the problem of branching water ways using uniform flow in all three channels at a junction as an initial condition. Other studies on transients in channel networks include those of Henry (1972), Debnath \& Chatterjee (1979), Chatterjee \& Debnath (1980), Kao (1980), Kan \& Yen (1981), Joliffe (1984), Gichuki et al (1990) and Swain \& Chin (1991). A common feature of all these studies is the consideration of simple channel junctions, at which continuity and energy equations form the boundary condition. However, modelling a canal network requires considerations of several types of control structures such as gates, weirs, pipe outlets, canal falls etc. These control structures may operate under free or submerged flow conditions.

This paper presents a generalised formulation for the analysis of transients in a canal network under subcritical flow, with any realistic combination of control structures and multiple operation of these structures. Figure 1 shows a schematic diagram of a 


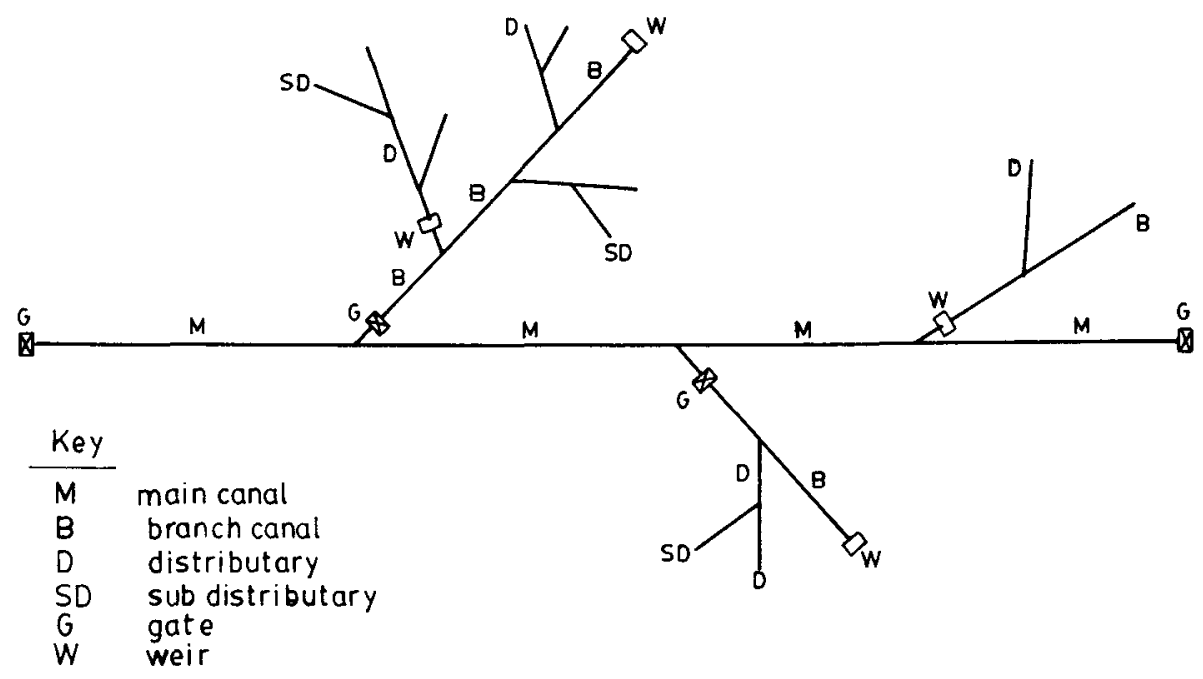

Figure 1. Schematic diagram of a canal network.

canal network with different types of control structures. These structures may be static, such as a weir and a canal fall, or dynamic, such as a moving gate, which may cause the transients. The present analysis is based on numerical solution of Saint-Venant equations using a characteristic method on a rectangular grid.

\section{Basic equations and methodology}

The Saint-Venant equations for one-dimensional gradually varied unsteady flow are given by

$$
\begin{aligned}
& A(\partial v / \partial x)+v T(\partial y / \partial x)+T(\partial y / \partial t)=0 \\
& (v / g)(\partial v / \partial x)+(\partial y / \partial x)+(1 / g)(\partial v / \partial t)=\left(S_{0}-S_{f}\right)
\end{aligned}
$$

Here $x$ is the distance along the channel, $t$ is the time, $y$ is the depth of flow, $v$ is the velocity of flow, $A$ is the cross-sectional area, $T$ is the top width, $S_{0}$ is the bed slope, $S_{f}$ is the friction slope and $g$ is the acceleration due to gravity. $S_{f}$ is given by Manning's equation as,

$$
S_{f}=n^{2} v^{2} / R^{4 / 3}
$$

Here $n$ is Manning's roughness coefficient and $R$ is the hydraulic radius.

Equations (1) and (2) can be solved using explicit, implicit or characteristic methods. Price (1974) compared the characteristic method on a rectangular grid with other explicit and implicit methods, and concluded that it was the most accurate one. Moreover, direct explicit methods are inflexible. The advantage of implicit methods in selecting a large time step may be more suitable for flood routing problems, where simulation has to be done for a very large time. But for transient analysis in a canal network, where unsteady flow changes are far more rapid as compared to floods, a 
small time step has to be chosen to get good accuracy. Besides, the method of characteristics can distinctly capture any sudden or sharp fluctuations that may occur due to transients.

Along the characteristics

$$
(\mathrm{d} x / \mathrm{d} t)=v \pm c
$$

(1) and (2) reduce to

$$
(\mathrm{d} v / \mathrm{d} t) \pm(g / c)(\mathrm{d} y / \mathrm{d} t)=g\left(S_{0}-S_{f}\right)
$$

where $c$ is the celerity of a gravity wave and is given by

$$
c=(g A / T)^{1 / 2} \text {. }
$$

The first of (5) is valid along the first of (4), which is referred to as forward characteristic, and the second of (5) is valid along the second of (4), which is referred to as backward characteristic.

\section{Types of boundaries}

Boundaries which commonly occur in a canal network can broadly be grouped into four types, namely, no-control structure, non-transient control structure, transient control structure and hydrograph.

\subsection{No-control structure}

The energy equation at a simple junction with no-control structures is written as

$$
\left(v_{u}^{2} / 2 g\right)+y_{u}-\left(v_{d}^{2} / 2 g\right)-y_{d}=H_{f} .
$$

Here $H_{f}$ is the junction head loss for which a suitable empirical relation can be used or alternatively the energy loss can be ignored. $y_{u}$ and $v_{u}$ are the depth and velocity at a node upstream to a junction whereas $y_{d}$ and $v_{d}$ are the depth and velocity at a node downstream to a junction.

\subsection{Non-transient control structures}

The discharge equation of any control structure which does not depend on time is grouped under this category. This type of control structure can be a weir, notch, spillway without gate or a rating curve. The general form of equation for this type of control structure can be written as

for all

$$
\begin{aligned}
& y_{u}>p_{u}(\text { figures } 2 \mathrm{a} \text { and } \mathrm{b}), \\
& Q=A v=k f\left(y_{u}, y_{d}\right),
\end{aligned}
$$

and for all

$$
\begin{aligned}
& y_{u} \leqslant P_{u} \text { (figure } 2 \mathrm{c} \text { ), } \\
& v=0 .
\end{aligned}
$$


(a)

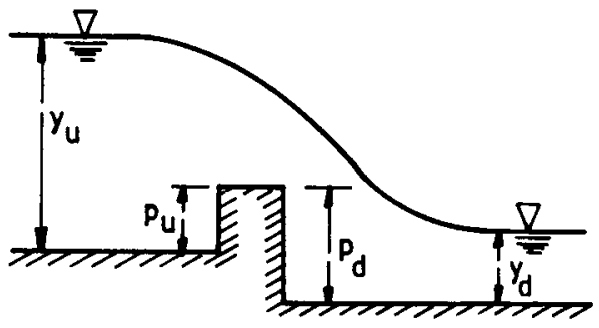

(b)

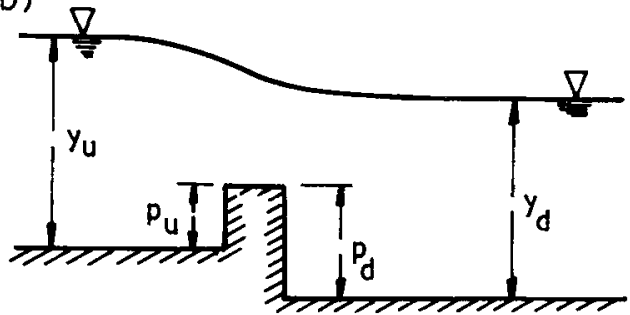

(c)

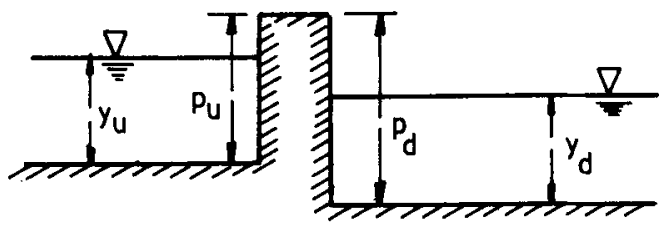

Figure 2. Flow over a non-transient control structure (a weir).

Here $Q$ is the discharge from the control structure and $k$ is the proportionality constant which is a function of the coefficient of discharge and the dimensions of the structure. The rating curve relation in the form of $Q=f(y)$ can be treated as a special case of (8a).

\subsection{Transient control structures}

The discharge equation at any control structure which depends on time (such as a sluice gate), is grouped under this category. The general form of governing equation for this type of control structure can be written as for all

for all

$$
\begin{aligned}
& y_{u}>\left(p_{u}+g_{t}\right)(\text { figures } 3 \mathrm{a} \text { and } \mathrm{b}), \\
& Q=A v=k f\left(y_{u}, y_{d}, g_{t}\right),
\end{aligned}
$$

$$
\begin{aligned}
& p_{u}<y_{u} \leqslant\left(p_{u}+g_{t}\right)(\text { figure } 3 c), \\
& \left(v_{u}^{2} / 2 g\right)+y_{u}-\left(v_{d}^{2} / 2 g\right)-y_{d}=H_{f g},
\end{aligned}
$$

and for all

$$
\begin{aligned}
y_{u} & \leqslant p_{u}(\text { figure } 3 \mathrm{~d}), \\
v & =0 .
\end{aligned}
$$


(a)

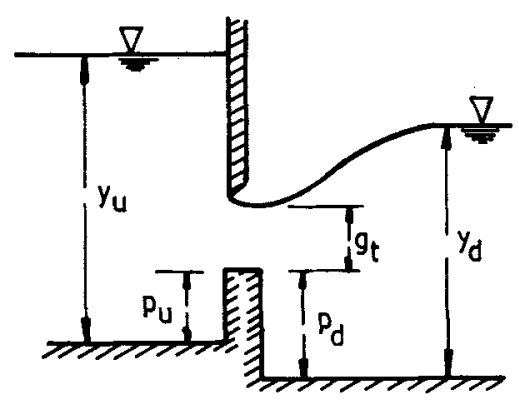

(c)

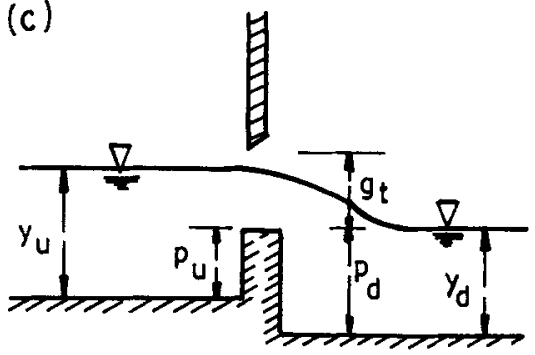

(b)

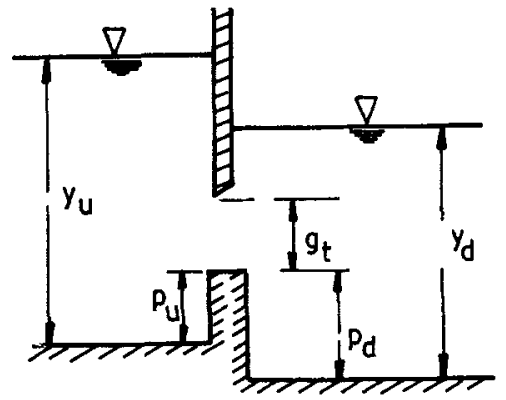

(d)

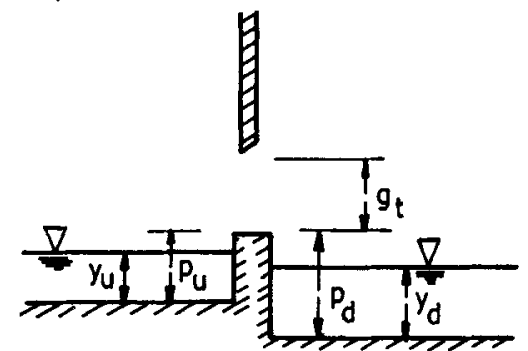

Figure 3. Flow through a transient control structure (a sluice gate).

Here $p$ is the height of the gate sill above the channel bed, $g_{t}$ is the opening of the gate at any time $t$, and $H_{f g}$ is the head loss due to flow over the sill when the water surface is entirely below the gate.

The modular limits of the control structure decide the submerged (figure $2 b, 3 b$ ) and unsubmerged (figures $2 \mathrm{a}, 3 \mathrm{a}$ ) flow condition and the value of coefficient of discharge to be used (Bos 1979).

\subsection{Hydrographs}

Observed state and discharge hydrographs are examples of such boundary conditions. The general form of the governing equations at such boundaries can be written as

or

$$
y=f(t)
$$

$$
Q=f(t)
$$

Usually hydrographs are not specified for canal networks.

\section{Boundary conditions}

In a canal network, the ends of extreme canals which do not intersect any other canal form an external boundary, referred to here as network boundary while the canal intersections known as junctions are referred to as internal boundaries. 


\subsection{Network boundary condition}

The non-transient or transient control structures or a location with specified hydrograph can form network boundaries. Network boundary nodes are assumed to lie at a sufficient distance from the physical location of the control structure so that the local disturbances in flow, like hydraulic jump below the gate etc. are stabilised.

\subsection{Internal boundary conditions}

The internal boundary conditions, also referred to as compatibility conditions, define the interrelationships at various junction nodes. These are normally a set of boundary equations which have to be satisfied simultaneously at any given junction. In the present study, the canal junction consists of one node per canal joining the junction. At all junctions, the junction continuity equation, written as

$$
\sum_{i=1}^{N} Q_{i}-\frac{\mathrm{d} S}{\mathrm{~d} t}=\sum_{i=1}^{N}(A v)_{i}-\frac{\mathrm{d} S}{\mathrm{~d} t}=0
$$

has to be satisfied. Here $N$ is the number of canals joining at the junction and $\mathrm{d} S / \mathrm{d} t$ represents the junction storage which can be ignored for a canal network. Apart from the junction continuity equation, $(N-1)$ additional equations are to be specified as boundary conditions, such as those given in (7) to (10).

Different combinations of layout and boundaries can occur at any channel junction. The set of equations for a channel junction can be classified under three types of compatibility conditions for subcritical flows, as follows.

Type 1: No-control structure at all canals joining at a junction (figure 4a). Compatibility conditions constitute one junction continuity equation, (11), and $(N-1)$ independent energy equations for a junction of $N$ canals.

Type 2: All channels, except the upstream channel, have a control structure (figure 4b). Compatibility conditions constitute one junction continuity equation, (11), and $(N-1)$ control structure equations for a junction of $N$ canals.

Type 3: A few canals have a control structure and a few (more than one) have no control structures (figure $4 \mathrm{c}$ ). Compatibility conditions are a set of one junction continuity equation, (11), $M$ control structure equations for $M$ channels with control structures, (8)-(10), and $(N-M-1)$ energy equations of the form of (7).

\section{Initial condition}

Usually the steady state flow solution is taken as initial condition for unsteady flow problems. The gauge readings under steady state of flow are usually not available for a canal network. The gradually varied flow profiles cannot be easily determined for the network as a whole due to unknown discharge distributions.

For a rising wave occurring due to gate opening, the initial discharge is generally low and hence any small errors in the specification of initial conditions is rapidly overridden by the relatively large unsteady flow changes due to the wave. For such a case, the initial condition is specified in the present study by obtaining the 
(a)
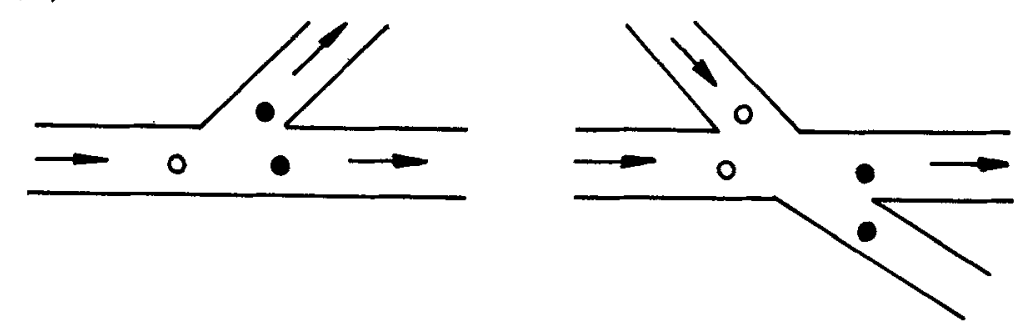

(b)
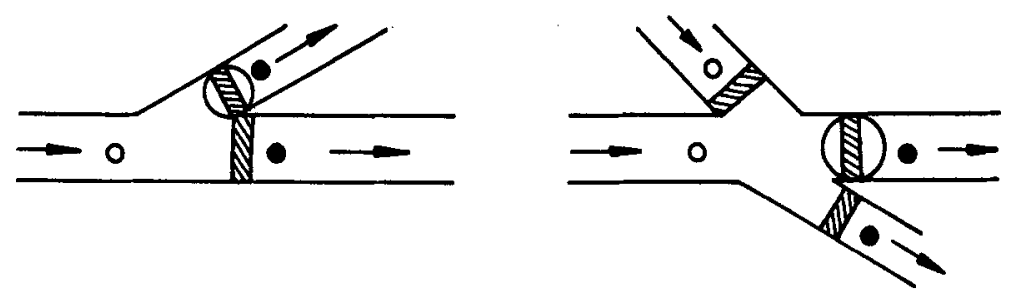

(c)
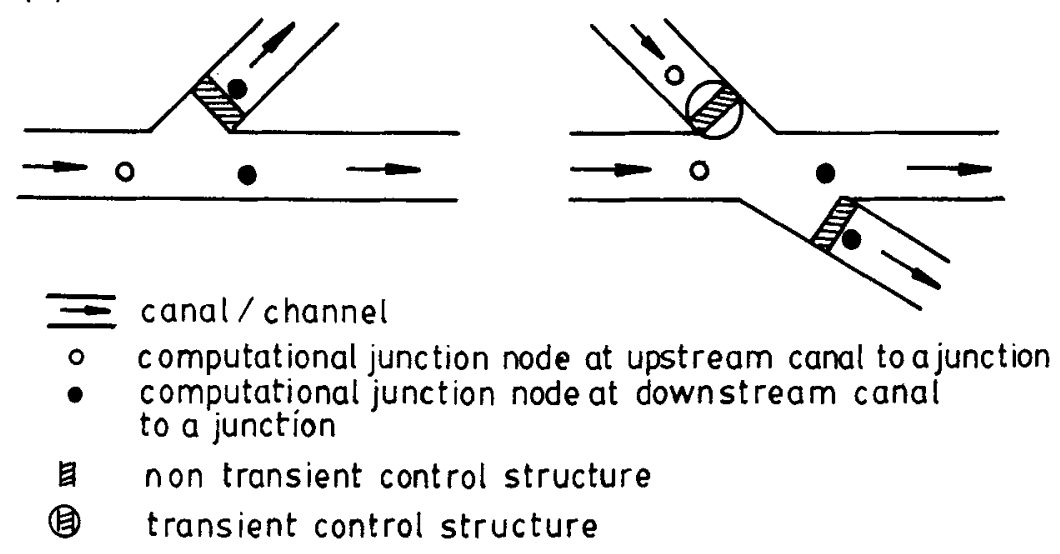

Figure 4. Different types of canal junctions.

approximate discharge distribution using uniform flow equation at nodes downstream of the junction and the compatibility conditions at the junction. With this discharge distribution, gradually varied flow computations are done to obtain $v$ and $y$ at all the nodes. The present unsteady flow algorithm can also be used to develop the steady state solution by simulation of flow for a sufficiently long period. This principle is used to specify the initial condition for cases where the initial discharge is significant such as in gate closure problems.

\section{Computational procedure}

The model uses the characteristic method on a rectangular grid with the spaceline interpolation scheme bounded by the Courant stability criterion. However, Koren's stability criterion (Huang \& Song 1985) is relaxed by taking friction terms and coefficients in (4) and (5) semi-implicitly. The numbering scheme for canals, junctions 
(a)

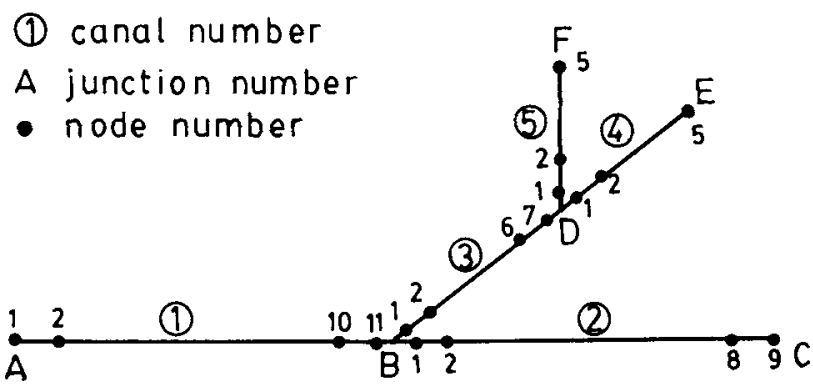

(b)

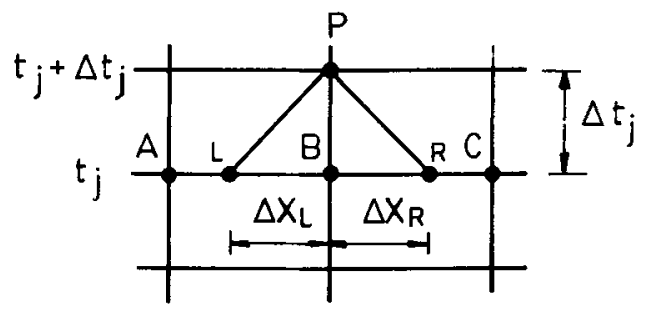

Figure 5. Definition sketch for discretisation and computational scheme.

and nodes is shown in figure 5, with a definition sketch for the finite difference grid as well. At any interior node, the finite difference forms of (4) and (5) are solved for four unknowns namely, $v_{p}, y_{p}, \Delta x_{L}$ and $\Delta x_{R}$ (figure $5 \mathrm{~b}$ ). The finite difference form of backward characteristic equations along with the network boundary condition are solved at all upstream network boundary nodes for unknown $v_{p}, y_{p}$ and $\Delta x_{R}$. At all downstream network boundary nodes, the finite difference form of forward characteristic equations along with the network boundary condition are solved to get $v_{p}, y_{p}$ and $\Delta x_{L}$. At an internal boundary such as a canal junction of $N$ canals, $2 N$ equations of relevant characteristics in finite difference form are solved along with $N$ boundary condition equations for $3 N$ unknowns, namely, $N$ number of $v$ values, $N$ number of $y$ values and $N$ number of $\Delta x_{L}$ or $\Delta x_{R}$ values.

Both finite difference equations and boundary condition equations are nonlinear. These sets of nonlinear equations at interior, network boundary as well as junction nodes, are solved iteratively using Newton's method coupled with under or over relaxation techniques, where required. The convergence is improved by starting the iteration with damped linearly extrapolated values of unknowns.

The time step $\Delta t$ is chosen dynamically in the computations such that the Courant condition is always satisfied and an unduly small $\Delta t$ value is not chosen. The $\Delta t$ value at any time satisfies the condition

$$
0 \cdot 8[\Delta x /(|v|+c)]_{\min } \leqslant t \leqslant 0 \cdot 95[\Delta x /(|v|+c)]_{\min } .
$$

\section{Application}

\subsection{Problem specification}

The present model has been used to simulate unsteady flows in a large variety of problems. To demonstrate the application of the proposed model, a simple irrigation 
canal nework with five canals, two junctions and four network boundaries has been selected (figure 5a). The geometric elements of all the five canals are given in table 1 . The transient effects due to simultaneous operation of gates with a time lag, at head-works (at $A$ in figure 5a), at second level canal (at $B$ in canal 3 in figure 5a) and at third level canal (at D in canal 5 in figure $5 \mathrm{a}$ ) are studied. There is a constant head reservoir, with a reservoir head of $3.5 \mathrm{~m}$, upstream of the gate at $A$ (figure 5). The width of the gate is taken as $6.5 \mathrm{~m}$ and coefficient of discharge is taken as 0.64 . The gate is assumed to function under free flow condition. The width of the gate at $B$ in canal 3 is taken to be $3.5 \mathrm{~m}$ and the coefficient of discharge is assumed to be 0.63 . The gate is assumed to flow under submerged conditions. The width of the gate in canal 5 at $\mathrm{D}$ is taken as $1.2 \mathrm{~m}$, the coefficient of discharge as 0.64 and the gate is assumed to discharge under submerged conditions. The heights of the gate sills at $B$ and D are taken as zero. Uniform flow boundary has been assumed at locations $\mathrm{C}$ and $\mathrm{E}$, whereas a rectangular finite crested weir of $1.2 \mathrm{~m}$ width and $0.3 \mathrm{~m}$ crest length in the direction of flow has been taken as the control boundary at $F$. The height of the weir ( $p_{u}$ in figure $2 \mathrm{a}$ ) is taken to be $0.15 \mathrm{~m}$. The coefficient of discharge is taken as constant for the broad crested range of operation, increasing linearly with $h / L$ ( $h$ is the head over the weir and $L$ is the crest length) in the narrow crested range, and then constant again for the sharp crested range at high heads (Ramakrishnan 1979).

The gate at $\mathrm{A}$ is opened from $0.1 \mathrm{~m}$ at $t=0 \mathrm{~s}$ to $1.2 \mathrm{~m}$ in $900 \mathrm{~s}$. The gate at $\mathrm{B}$ is opened from $0.1 \mathrm{~m}$ to $1.2 \mathrm{~m}$ in $500 \mathrm{~s}$, with the gate movement starting at $600 \mathrm{~s}$. The gate at $\mathrm{D}$ is opened from $0.1 \mathrm{~m}$ to $1.0 \mathrm{~m}$ in $300 \mathrm{~s}$, with the gate movement starting at $1000 \mathrm{~s}$. This problem has been chosen with a view to show that large and sudden fluctuations in discharges and depths may occur due to combined operation of gates in a canal system.

\subsection{Discussion of results}

Figures 6 to 13 present depth and discharge hydrographs at the first, middle and last nodes of the respective canals. Figure 14 presents the water surface profile along canal path 1-3-5 (figure 5a).

The present problem deals with the study of transient effects in the canal network due to three different direct waves originating at different locations. First, the gate at head-works $A$ is opened gradualiy and, before the wave reaches the junction $B$, the gate at $B$ starts opening. The opening of gate at D is also initiated before the earlier direct waves arrive at D. Because of these early openings of gates at B and D, the flow takes place below the gate for sometime and when the direct waves from upstream arrive, the water level rises above the bottom edge of the gate. Due to these complex flow conditions, large and rapid changes in flow depth and discharge occur in the canals. With a view to facilitate a discussion of flow changes, the important features of the hydrographs are marked by labels 1, 2, 3 etc. in figures 6 to 13 and are referred in the following discussions.

7.2a Effect of gate opening: Because of the gate opening at $\mathrm{A}$, the discharge in canal 1 at A rises from 3.36 cumecs $\left(\mathrm{m}^{3} / \mathrm{s}\right)$ to 36.05 cumecs in $900 \mathrm{~s}$ and remains fixed thereafter (figure 7). The gate on canal 3 at B starts opening at $600 \mathrm{~s}$ even before the direct wave from $A$ arrives at $B$ (at $1300 \mathrm{~s}$, figures 6 and 8). Hence the depth of flow in canals 1 and 2 at $B$ starts decreasing after $600 \mathrm{~s}$, as can be seen from 1 and 11 in figures 6 and 8 respectively. Due to this decreasing depth at $B$, the discharge in canal 


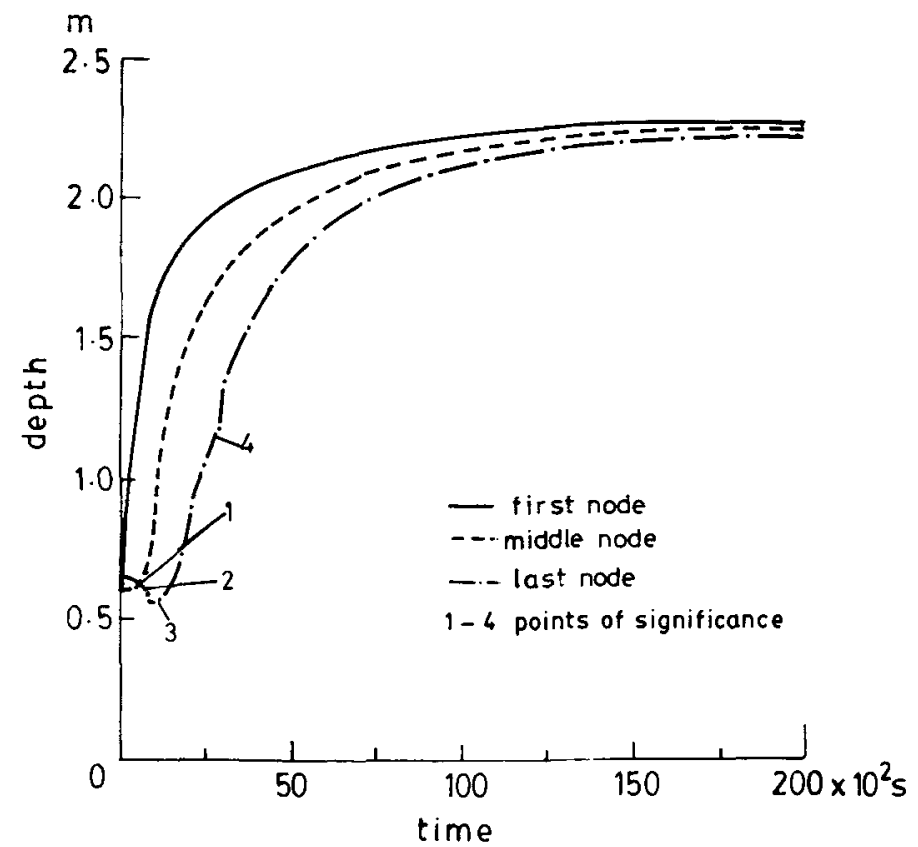

Figure 6. Depth hydrograph for canal 1.

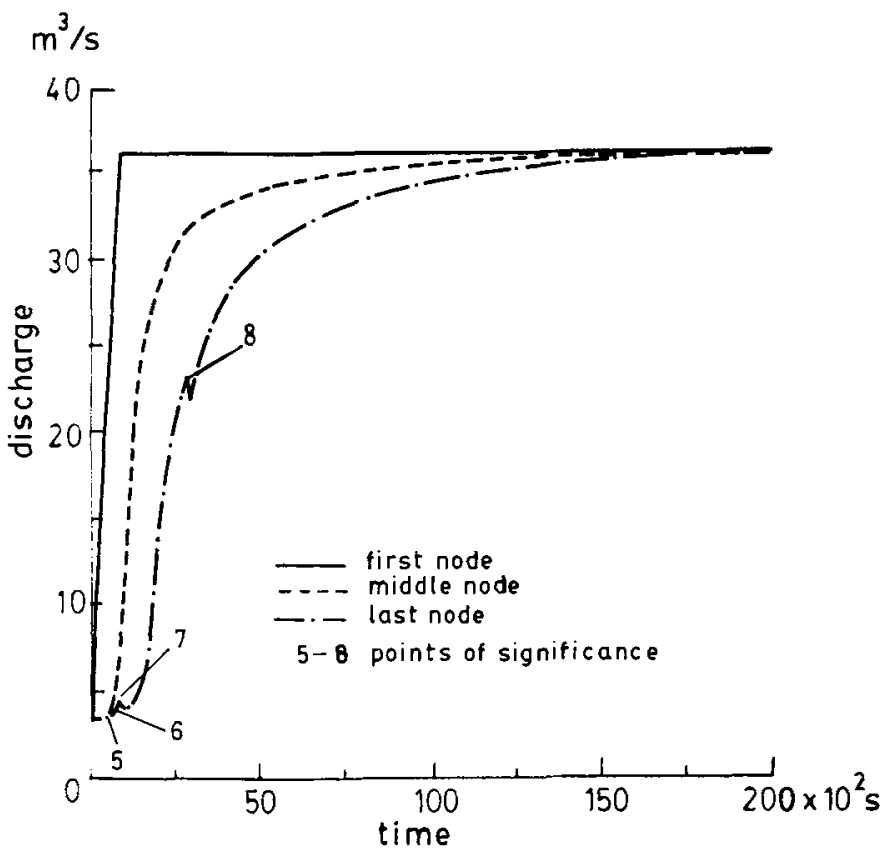

Figure 7. Discharge hydrograph for canal 1. 


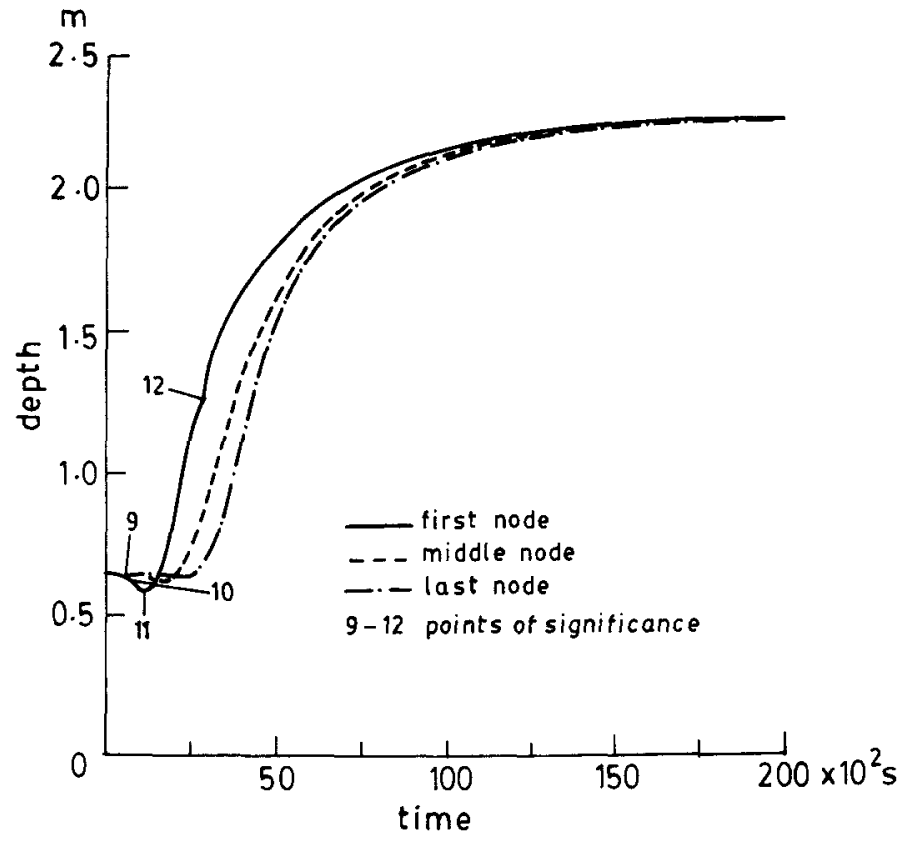

Figure 8. Depth hydrograph for canal 2.

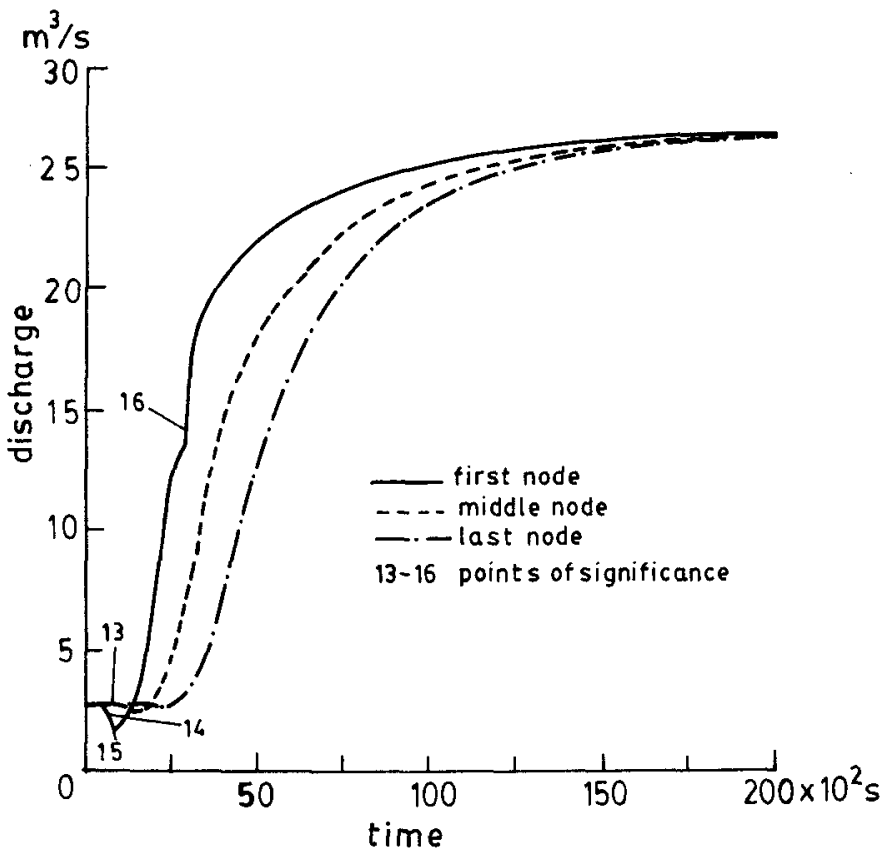

Figure 9. Discharge hydrograph for canal 2. 


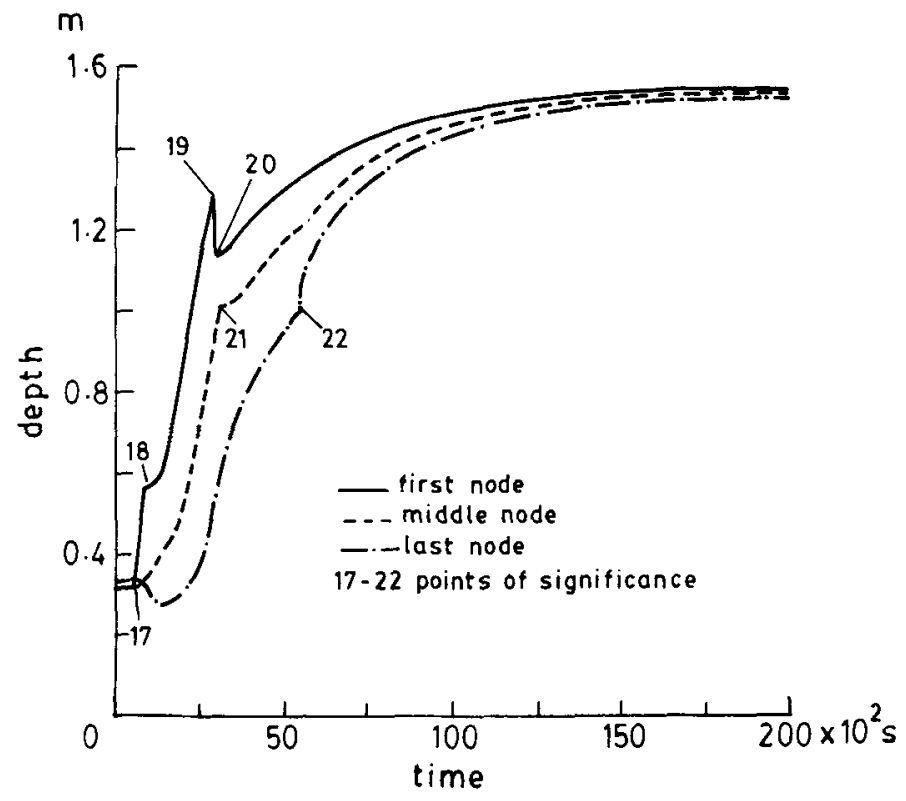

Figure 10. Depth hydrograph for canal 3.

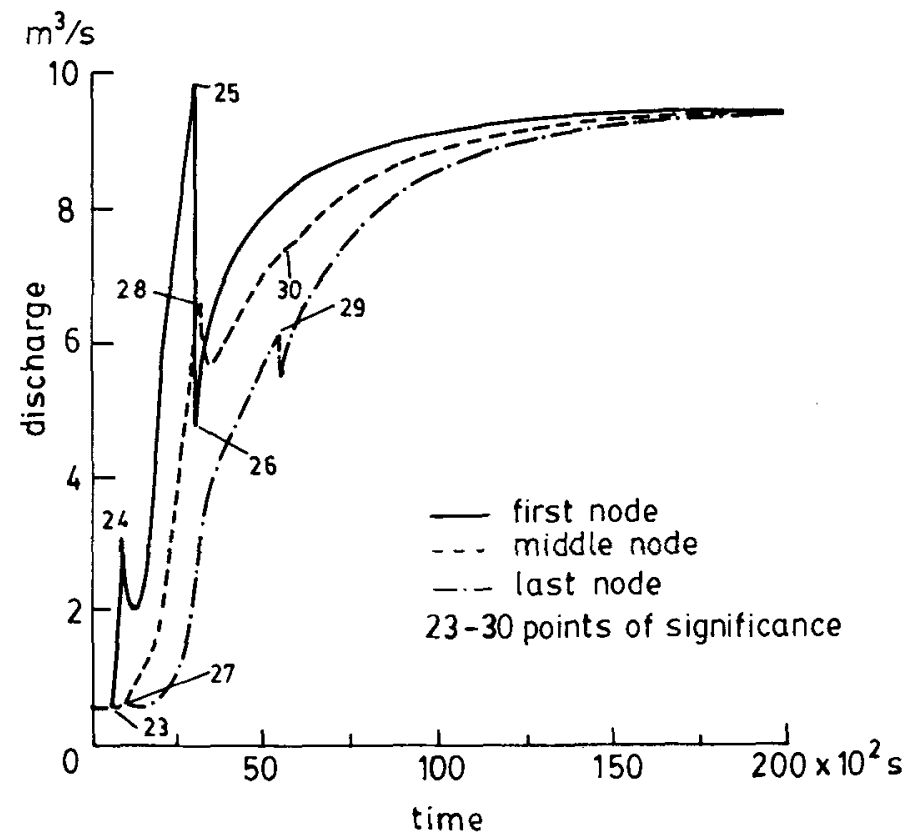

Figure 11. Discharge hydrograph for canal 3. 


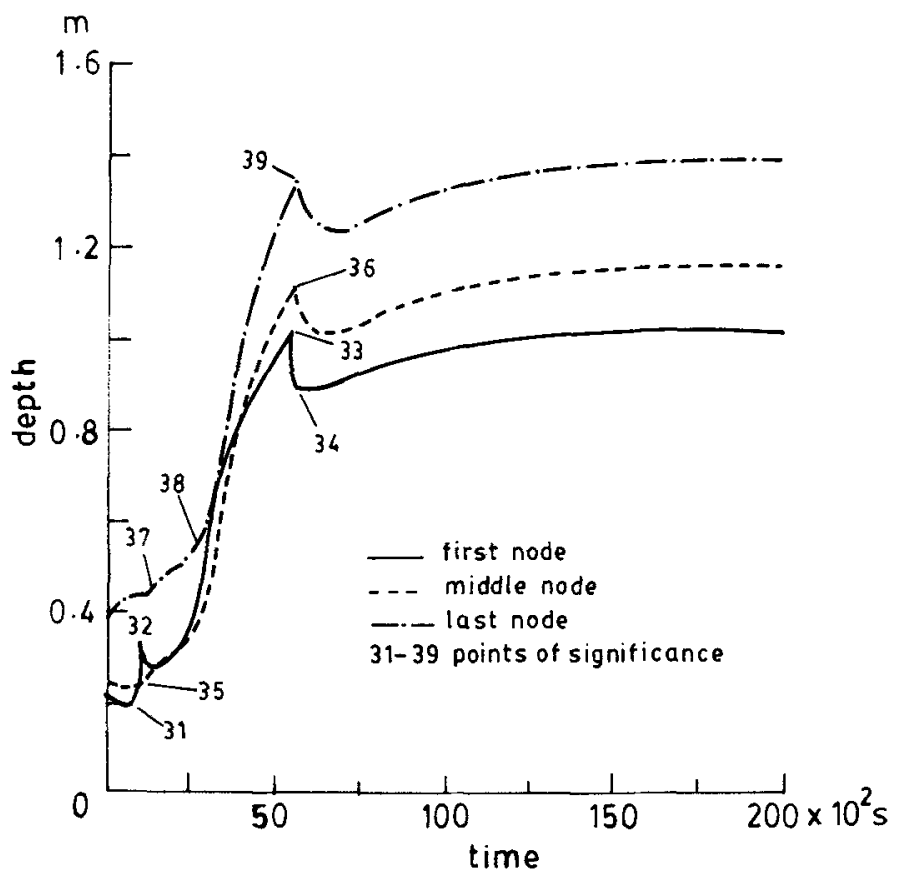

Figure 12. Depth hydrograph for canal 5.

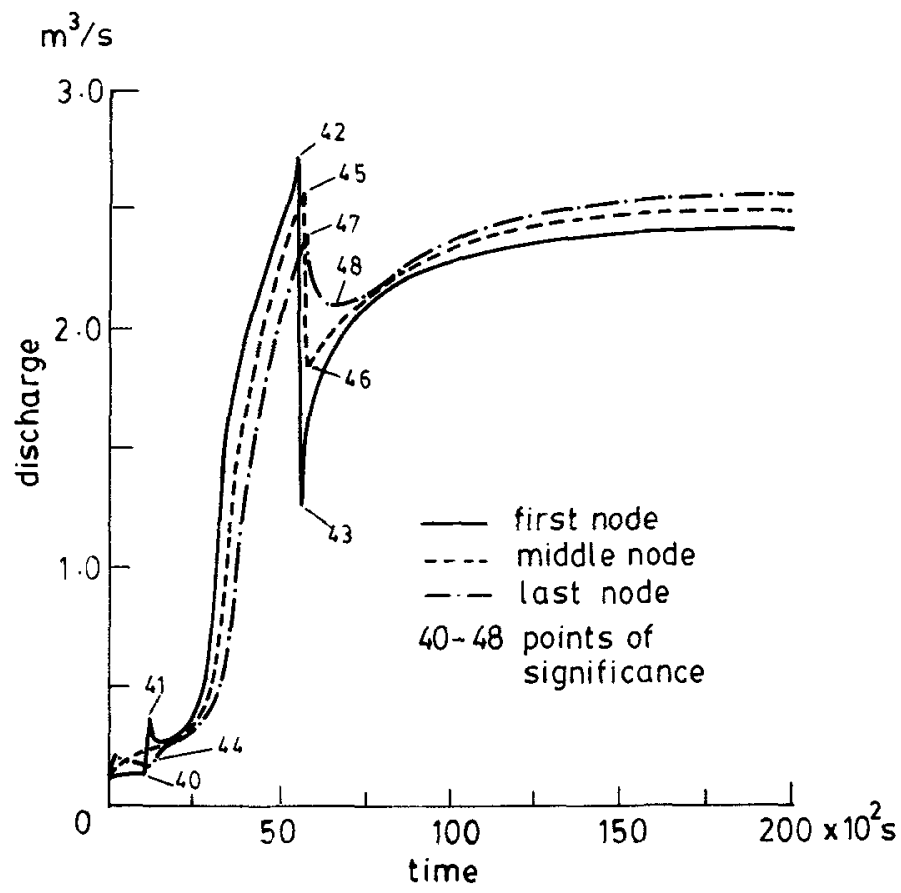

Figure 13. Discharge hydrograph for canal 5 . 


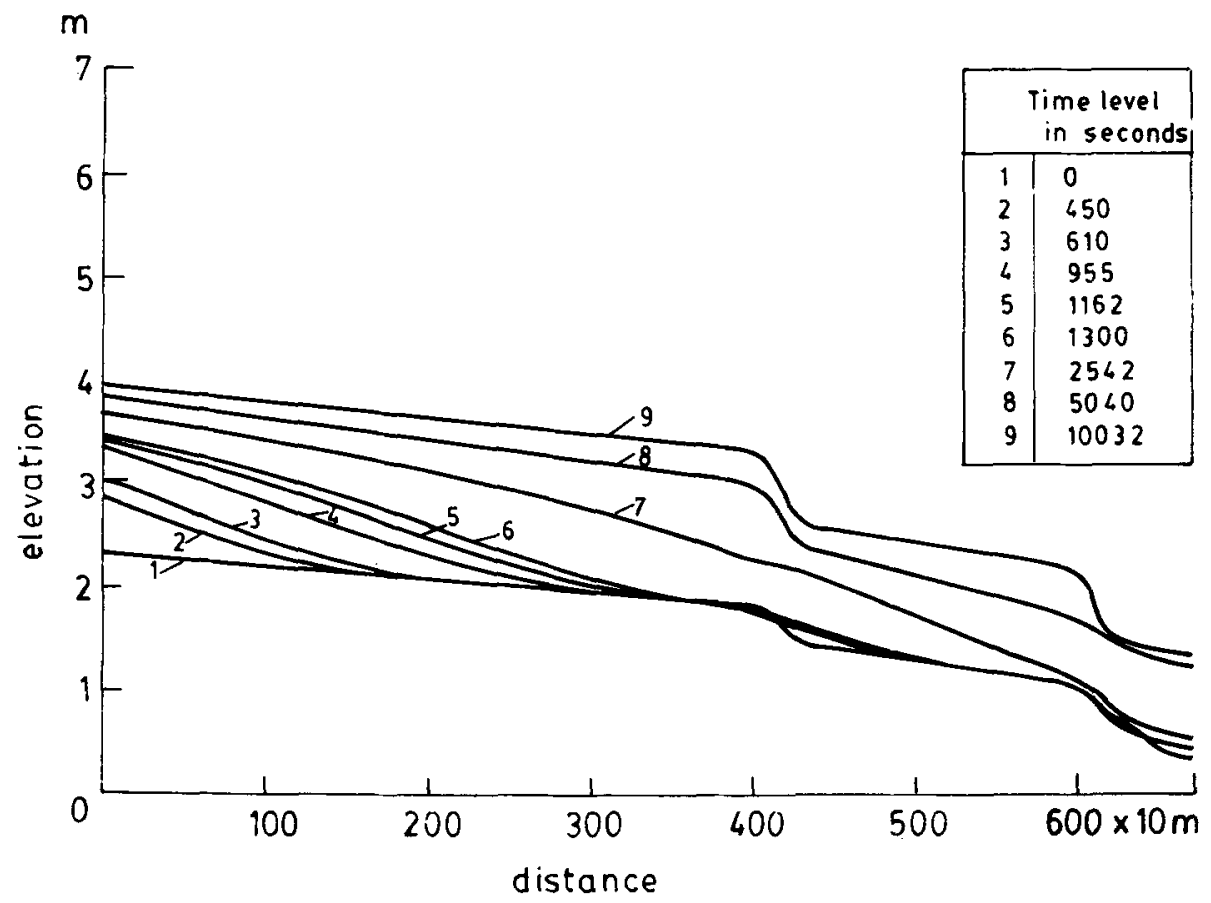

Figure 14. Water surface profile along 1-3-5 canal.

1 at $B$ starts increasing ( 5 to 7 in figure 7 ) and in canal 2 at $B$ starts decreasing (13 in figure 9) due to increasing and decreasing hydraulic gradients respectively. Because of the gate opening, the depth and discharge in canal 3 at B starts increasing after $600 \mathrm{~s}(17-18$ in figure 10 and $23-24$ in figure 11).

At $820 \mathrm{~s}$, the flow depth and gate opening at B both become equal to $0.584 \mathrm{~m} \mathrm{(3}$ in figure 6), and thereafter the water surface remains below the gate for some duration. Hence from $820 \mathrm{~s}$, the gate equation, (9), is replaced by the energy equation, (7), at junction $B$ in the computations. This change in the boundary condition at $B$ is reflected by changes in depth and discharge hydrographs in canal 1 (3 in figure 6 and 7 in figure 7), canal 2 (11 in figure 8 and 15 in figure 9) and canal 3 (18 in figure 10 and 24 in figure 11).

7.2b Wave effects at junctions: The gate at B gets fixed to its final opening of $1.2 \mathrm{~m}$ by $1100 \mathrm{~s}$. After the arrival of the direct wave from A at B about $1300 \mathrm{~s}$, the depth and discharge in canals 1,2 , and 3 at $\mathbf{B}$ start increasing as can be seen from figures 6-11. It should be noted here that water is still flowing below the gate at B. Upto $2850 \mathrm{~s}$, the depth of flow in canal 1 at $B$ increases gradually but remains less than $1.2 \mathrm{~m}$, the fixed gate opening (figure 6). At $2850 \mathrm{~s}$, the depth in canal 1 at B becomes equal to the gate opening $(1.2 \mathrm{~m})$ and the wave strikes the gate. The flow conditions at the junction again change and are governed by the gate discharge equation. This change occurring at $2850 \mathrm{~s}$, increases sharply the depth of flow at B in canal 1 (4 in figure 6 ) and in canal 2 (12 in figure 8 ) due to the obstruction posed by the gate. This sharp rise in depth upstream of the gate at $B$, reduces the discharge sharply in canal 1 due to reduced hydraulic gradient ( 8 in figure 7), whereas it increases the discharge sharply in canal 2 due to increased hydraulic gradient (16 in figure 9). As the water surface rises above the gate, the depth and discharge reduce very sharply downstream 
of the gate in canal 3 at B (19 to 20 in figure 10 and 25 to 26 in figure 11). The changes at $B$ are dissipated and/or overridden by the strong effect of the direct wave from canal 1 . Hence, the depth and discharge again start increasing at B in canals 1 , 2 and 3 (figures 6 to 11 ).

These changes in depth and discharge at $B$ travel upstream in canal 1 and downstream in canals 2 and 3 and get attenuated and the effects are seen particularly in canal 3 (21 in figure 10 and 28 in figure 11).

The gate at junction $D$ in canal 5 starts opening at $1000 \mathrm{~s}$. In the absence of the arrival of direct waves from $A$ and $B$, the flow goes below the gate upto $1600 \mathrm{~s}$. The gate is fully opened to $1.0 \mathrm{~m}$ at $1300 \mathrm{~s}$ and the water upstream of the gate rises to this level at $5300 \mathrm{~s}$. Thereafter, the discharge is governed by the gate equation. These features bring out more or less the same kind of changes in depths and discharges at junction D, as discussed earlier for junction B. This is seen in canal 3 (22 in figure 10 and 27, 29 and 30 in figure 11) and canal 5 (37, 38 and 39 in figure 12 and 40, 41, 42 and 43 in figure 13). The attenuated effects of changes occurring at $\mathrm{D}$ in canals 3,4 and 5 are shown by 21 in figure 10,30 in figure 11 and $44,45,46,47$ and 48 in figure 13.

The flow has reached final steady state at the end of the simulation time of $20,000 \mathrm{~s}$ as can be seen from figure 6-13. The wave/water surface profiles at different times as seen along the canal path 1-3-5 are shown in figure 14 .

\section{Conclusion}

A mathematical model is developed for the analysis of transients in a canal network due to the operation of control structures. The model considers different types of static and moving control structures occurring in different combinations at channel junctions and network boundaries. The model has been applied to a complex flow situation arising due to multiple gate operations in a network. It is also shown that the model can be used to simulate steady flows in a canal network for fixed positions of control structures.

\section{References}

Bos M G 1976 Discharge measurement structure (New Delhi: Oxford and IBH).

Chatterjee A K, Debnath L 1980 Mathematical model for flood and embankment prediction in a tidal river. Acta Mech. 36: 187-194

Debnath L, Chatterjee A K 1979 Nonlinear mathematical model for the propagation of tides in interlocking channels. Comput. Fluids 7: 1-12

Gichuki F N, Walker W R, Merkley G P 1990 Transient hydraulic model for simulating canal network operation. J. Irrig. Drain. Eng., ASCE 116(1): 67-82

Henry R F 1972 Simulation of long waves in branching water ways. J. Hydraul. ASCE 89: 604-629

Huang J, Song C C S 1985 Stability of dynamic flood routing schemes. J. Hydraul. ASCE 111: 1497-1505

Joliffe I B 1984 Computations of dynamic waves in channel networks. J. Hydraul. ASCE 110: 1358-1370

Kan A O A, Yen B C 1981 Diffusion wave flood routing in channel network. J. Hydraul. ASCE 107: 719-732

Kao J E 1980 Improved Implicit procedure for multichannel surge computations. Can. J. Civil Eng. 7: $502-512$

Price R K 1974 Comparison of four numerical methods for flood routing. J. Hydraul. ASCE 100: 879-899

Ramakrishnan C R 1979 Flow characteristics of rectangular and trapezoidal finite crested weirs and triangular profile weirs, $\mathrm{PhD}$ thesis, Indian Institue of Science, Bangalore

Stoker J J 1957 Water waves (New York: Interscience)

Swain E D, Chin D A 1991 Model of flow in regulated open channel network. J. Irrig. Drain. Eng. ASCE 116: $537-556$ 